\title{
HNSCC: Depression verkürzt das Leben
}

Patienten mit Plattenepithelkarzinom im Kopf- und Halsbereich sind häufiger von Depressionen betroffen als andere Tumorpatienten. Wie stark beeinflusst eine Depression die Prognose?

erade Patienten mit neu diagnostiziertem Plattenepithelkarzinom im Kopf-Hals-Bereich (HNSCC) haben ein hohes Risiko für eine Depression oder eine depressive Verstimmung. Diese wirkt sich negativ auf die Lebensqualität und den Ernährungsstatus nach der Therapie aus. Fehlernährung ist bei KopfHals-Tumoren verbunden mit erhöhter Morbidität und Mortalität. Depression könnten also das Überleben beeinflussen.

$\mathrm{Ob}$ dies zutrifft, wurde in einer prospektiven Studie mit 241 zuvor unbehandelten HNSCC-Patienten untersucht, die eine kurative Behandlung erhielten. Ihr psychischer Zustand vor Beginn der Therapie wurde mithilfe des Beck Depression Inventory (BDI) II evaluiert, die Lebensqualität mithilfe des EORTC QLQ C30-
Fragebogens und des EORTC QLQ Head and Neck Cancer-Module (QLQ-H\&N35). Patienten wurden ab einem BDI-II-Score von $\geq 14$ als depressiv eingestuft.

Vor Therapiebeginn lag bei 60 Patienten $(24,9 \%)$ eine Depression vor. Bei depressiven bzw. nicht depressiven Patienten betrug die Rate für das 3-JahresGesamtüberleben (OS) 70,8 bzw. 82,7\% ( $\mathrm{p}=0,045)$, für das krankheitsfreie Überleben (DFS) 63,5 bzw. 79,1\% (p=0,015). Nach Ausschluss klinischer Faktoren war eine Depression prädiktiv für das 3-Jahres-DFS ( $p=0,032)$.

Bei fast allen abgefragten Faktoren zur Lebensqualität unterschieden sich die Werte von depressiven und nicht depressiven Patienten. Depressive Patienten hatten zudem niedrigere Serumal-

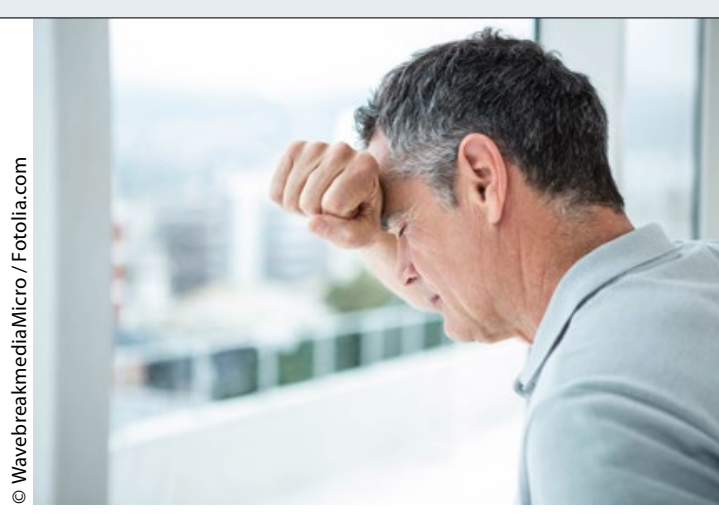

Eine Depression war prädiktiv für das krankheitsfreie 3-Jahres-Überleben.

buminspiegel vor der Behandlung Patienten ohne Depression $(\mathrm{p}<0,05)$.

Fazit: Eine Depression korreliert signifikant mit der Lebensqualität vor der Therapie, Ernährungsstatus und Überlebensdaten. $\mathrm{Ob}$ eine Depression vorliegt, lässt sich mittels BDI-II gut und einfach bestimmen. Depressiven Patienten könnte man dann frühzeitig eine Intervention anbieten.

Christina Berndt

Kim S-A et al. Pretreatment Depression As a Prognostic Indicator of Survival and Nutritional Status in Patients With Head and Neck Cancer. Cancer. 2016;122(1):131-40.

\section{Auch am Hals auf Wächterlymphknoten Verlass}

\begin{abstract}
Mit der Biopsie von Wächterlymphknoten lassen sich zuverlässig okkulte Metastasen am Hals von Patienten mit Mundhöhlenkarzinomen ausschließen. Allerdings ist die Rate falsch-negativer Ergebnisse grenzwertig hoch.
\end{abstract}

$R^{n}$ und jeder dritte Patient mit Mundhöhlenkarzinom im Stadium I/II hat radiologisch nicht nachweisbare zervikale Metastasen. Die Heilungschance sinkt dadurch um die Hälfte. Bei einem Metastasierungsrisiko von über $20 \%$ schlägt man den Patienten meist eine elektive Neck-Dissection vor, d.h. fast $80 \%$ unterziehen sich dieser Prozedur unnötigerweise. Die Entnahme von Wächterlymphknoten (SLN) wurde deshalb in der multizentrischen, prospektiven europäischen SENT-Studie getestet, deren 3-Jahres-Ergebnisse nun vorliegen. Bei den 415 Patienten hatte die ra- diologische Diagnostik einen Tumor im Stadium T1-2N0 ergeben. Nach Injektion eines Technetium-Nanokolloids ließ sich bei 99,5\% der Patienten ein SLN identifizieren. In 94 von 415 Fällen (23\%) war er positiv, die Betroffenen erhielten binnen 3 Wochen eine NeckDissection. 15 der übrigen 321 Patienten entwickelten bei negativer SLN-Pathologie und negativem Tumorbett zervikale Metastasen, d.h. die Falsch-NegativQuote betrug 14\% (15 von 109 Patienten mit okkulter zervikaler Metastasierung).

Für die SLN-Biopsie bei Mundhöhlenkarzinom ergaben sich eine Sensitivität von $86 \%$ und ein negativer Vorhersagewert von $95 \%$. Die Rate für das Gesamtüberleben betrug $88 \%$, für das krankheitsfreie Überleben $92 \%$ und für das krankheitsspezifische Überleben $94 \%$ jeweils nach 3 Jahren.

Den Hauptnutzen der SLN-Methode sehen die Forscher darin, dass $71 \%$ der Patienten eine Neck-Dissection erspart blieb. Allerdings liege die Rate falschnegativer Ergebnisse mit $14 \%$, an der Grenze des Akzeptablen“. Zwar ähnle die Rate jener, die von Melanompatienten bekannt sei, sie sei aber doppelt so hoch wie bei Patientinnen mit Mammakarzinom. „7\% sollten unser Ziel sein“.

Andererseits war nur bei 8 von 15 der fälschlich als nodal negativ eingestuften Patienten ein rettender Eingriff möglich, und mit der SLN-Methode konnten 7 positive Knoten kontralateral zur Tumorlage ausgemacht werden, die man bei der üblichen ipsilateralen Neck-Dissection nicht erkannt hätte.

Fazit: Die SLN-Biopsie ist auch bei Patienten ohne okkulte zervikale Lymphknotenmetastasen relativ zuverlässig. Noch lässt die Sensitivität zu wünschen übrig, aber sie könnte sich durch verbesserte Technik und mehr Erfahrung gesteigert werden.

Robert Bublak

Schilling $C$ et al. Sentinel European Node Trial (SENT): 3-year results of sentinel node biopsy in oral cancer. Eur J Cancer. 2015;51(18):2777-84. 